\title{
A statistical analysis of fatalities resulting from Boko Haram activities
}

\author{
Emmanuel Afuecheta ${ }^{1,3, *}$, Chigozie Utazi ${ }^{2}$ and Diego Andrés Pérez Ruiz ${ }^{3}$ \\ ${ }^{1,3}$ School of Mathematics, University of Manchester, Manchester M13 9PL, UK \\ ${ }^{2}$ Southampton Statistical Science Research Institute, University of Southampton, SO17 1BJ, UK
}

Received May 27, 2016; December 27, 2016; Published Online December 31, 2016

Copyright (C) 2016, African Journal of Applied Statistics (AJAS) and Probability African Society (SPAS). All rights reserved

\begin{abstract}
For the first time, a statistical analysis of the number of deaths due to Boko Haram activities is provided. The analysis is based on some newly proposed discrete distributions. The distributions are fitted to the data using the method of maximum likelihood. The discrete Chen distribution is found to give the best fit. Its parameters are allowed to vary linearly with time to capture the variability in the number of deaths resulting from Boko Haram attacks. Predictions are given in terms of possible future number of deaths. As a byproduct, some basic properties of the discrete Chen distribution are provided.

Résumé. Pour la première fois, une analyse statistique du nombre de décès dus aux activités de Boko Haram est fournie. L'analyse est basée sur des distributions discrètes nouvellement proposées. Les distributions sont adaptées aux données en utilisant la méthode du maximum de vraisemblance. La distribution discrète de Chen est trouvée pour donner le meilleur ajustement. Ses paramètres sont autorisés à varier linéairement avec le temps pour capturer la variabilité dans le nombre de décès résultant des attaques de Boko Haram. Les prédictions sont données en termes de nombre futur possible de décès. En tant que sous-produit, certaines propriétés fondamentales de la distribution discrète de Chen sont fournies.
\end{abstract}

Key words: Boko Haram; Discrete distributions; Number of deaths.

AMS 2010 Mathematics Subject Classification : 90B10; 97B10.

Presented by Professor Gane Samb Lo, Université Gaston Berger de Saint-Louis, Sénégal. Member of Editors Board.

\footnotetext{
${ }^{*}$ Corresponding author Emmanuel Afuecheta: emmanuel.afuecheta@manchester.ac.uk Chigozie Utazi : utazichigozie@yahoo.com

Diego Andrés Pérez Ruiz : rdiego.a.perez.r@gmail.com
} 
Afuecheta E., Utazi C. and Ruiz A. P. African Journal of Applied Statictics, Vol. 3 (1), 2016, 101 - 120. A statistical analysis of fatalities resulting from Boko Haram activities.

\section{INTRODUCTION}

Boko Haram, an islamic sect operating mostly in the northern part of Nigeria seems to have emerged after the 9/11 attack, and has become more violent since 2009. Continuously, from 2009 while advocating for the creation of islamic states ruled by sharia law, a number of people have lost their lives. Many churches have been attacked, as well as government buildings, the media, police stations, schools, and the UN office in the Abuja. Their attacks are undoubtedly increasing by the day and have been in the media spotlight across the globe. See ( Fig 5 ) for mostly affected areas. This poses a serious threat to Nigeria's unity, its economy and development. Also, it undermines public safety across the region and has tremendously decreased ethnic and religious tolerance ([Maiangwa and Uzodike (2012)]; [International Crisis Group (2014)]).

However, irrespective of government efforts to extirpate the group via multiple tracks such as increase in defence budget, anti-terrorism law, declaration of state of emergency and military offensive, Boko Haram has proved to be resilient while changing its tactics and targets at the precept of it's leader, Abubakar Shekau [Walker (2012)].

The recent audacious attacks and abduction of over 200 Chibok school girls have raised concerns and caused outrage across the country and beyond. According to Amnesty International, more than 5000 people have been killed in the first and two quarters of 2014 and over 12,000 civilians brutally displaced. More than 3000 houses have also been destroyed.

To this end, there have been a good number of scholarly papers on Boko Haram activities, centering mainly on questions surrounding the emergence of Boko Haram, such as islamic revivalism, poverty and good governance, frustration aggression, unemployment, social and political economic challenges (See, for example, [Onapajo and Uzodike (2012)]; [Thomson (2012)]; [Walker (2012)]; [Agbiboa (2014)] ). Also, the emergence of Boko Haram has political undertone and this concludes why politicians always hide in sect's violent dispositions to achieve their political and selfish interest [Adesoji (2010)]. It has also been observed that sustained economic hardship and rising inequality are encouraging the proliferation of radical groups like Boko Haram. They however, noted that nothing less than an overhaul of the country's political governance is needed to flat-foot the extremists [International Crisis Group (2014)].

Regrettably, none of these papers have explored the use of statistical techniques to give a panoramic view of the sect's menace. As such, this paper seeks to analyze the number of deaths/fatalities resulted from the Boko Haram's heinous activities. Our primary aim is to employ discrete distributions and statistical models based on those discrete distributions to characterize and extrapolate the overall number of deaths due to Boko Haram attacks in Nigeria. To the best of our knowledge, this is the first paper applying statistical methodology to study Boko Haram insurgency. The contents of this paper are organized as follows. Section 2 describes the data on the number of deaths across the country. Section 3 describes the various discrete distributions used. The results and their discussion are provided in Section 4. Basic distributional properties of the discrete Chen distribution are provided in Section 5. Finally, some conclusions are given in Section 6 .

\section{Data}

The data are maximum monthly number of deaths from January 2009 to 1st July, 2014 and yearly number of attacks by target types. The data were extracted from two websites:

Journal home page: www.jafristatap.net 
Afuecheta E., Utazi C. and Ruiz A. P. African Journal of Applied Statictics, Vol. 3 (1), 2016, 101 - 120. A statistical analysis of fatalities resulting from Boko Haram activities.

the first, www.start.umd.edu/gtd/, a global terrorism database which keeps track of all information on terrorist events around the world and the second, factsnigeriaviolence.org, a blog giving daily maximum number of deaths and attacks by target types (Christian community, muslim community, government structures, schools, media/medical, others) across the states (Anambra, Enugu, Akwa Ibom, Adamawa, Abia, Bauchi, Bayelsa, Benue, Borno, Cross River, Delta, Ebonyi, Edo, Ekiti, Gombe, Imo, Jigawa, Kaduna, Kano, Katsina, Kebbi, Kogi, Kwara, Lagos, Niger Nasarawa, Ogun, Ondo, Osun, Oyo, Plateau, Rivers, Sokoto, Taraba, Yobe, Zamfara) in Nigeria. However, in our statistical analysis, we only used maximum number of deaths between January 2010-1st July,2014. This is to avoid the occurrence of too many zeros in our data since there were quite a few number of attacks and deaths before 2010 .

Fig 1 shows the distribution of the number of deaths per years. The number increases by year and appears largest for 2014 and second largest for 2013 in terms of median and and spread. It appears smallest for the years 2009 and 2010. Fig 2 shows the distribution of the number of attacks versus target types (Christian community, muslim community, government structures, schools, media/medical, others). The christian community receives the largest number of attacks, government structures receives the second largest number of attacks in terms of median and spread. The media/medical, muslim and schools receive the least number of attacks in that order. This confirms that of all the factors promoting the groups's activities, religion still stands out as the most prominent. Fig 3 shows the distribution of the number of attacks versus years. In terms of median and variability, the number of attacks appear largest in 2010, and second largest in 2014. The number of attacks appear smallest in 2013 and 2011. Fig 4 shows a scatter plot of number of deaths versus months. A visual inspection of it shows an increasing trend in the number of deaths due to attacks with pronounced variation at the tail end of the study period. Observe that in Fig 1 and Fig 3, the distributions of the number of deaths and attacks in 2010 and 2014 respectively differ significantly in terms of median and variability. This is evidence of Boko Haram becoming more sophisticated, coordinated, menacingly daring and seemingly genocidal in its attacks. Launching of serial and multiple offensive operations at high profile places like markets, banks, drinking spots and bus stations [Aghedo and Osumah ((2012)].

\section{Models}

Discrete frequency distributions often involve count of occurrences. Characterization of such outcomes therefore requires suitable probability mass functions. Here we give a description of eleven discrete probability distributions used, each with its own properties making it unique and as such distinguishing it from others. Seven of the distributions (Dagum, Shabe, loglog, log-logistic, inverse exponentiated exponential, Nakagami,and Chen) are being proposed for the first time, while three (lognormal,generalized pareto, Frechet) were proposed by [Nadarajah (2013)] and one (Poisson) is a common one, often used in modelling count data. However, our choice of distributions include both light and heavy-tailed distributions. This is to account for some features ( if any ) such as outliers, overdispersion, underdispersion which are common with count data relative to some discrete distributions. The light-tailed distributions are log normal, Nakagami, poisson, inverse exponentiated exponential and Schabe, while the heavy-tailed distributions are Dagum, generalized pareto, Frechet and log-logistic.

Journal home page: www.jafristatap.net 


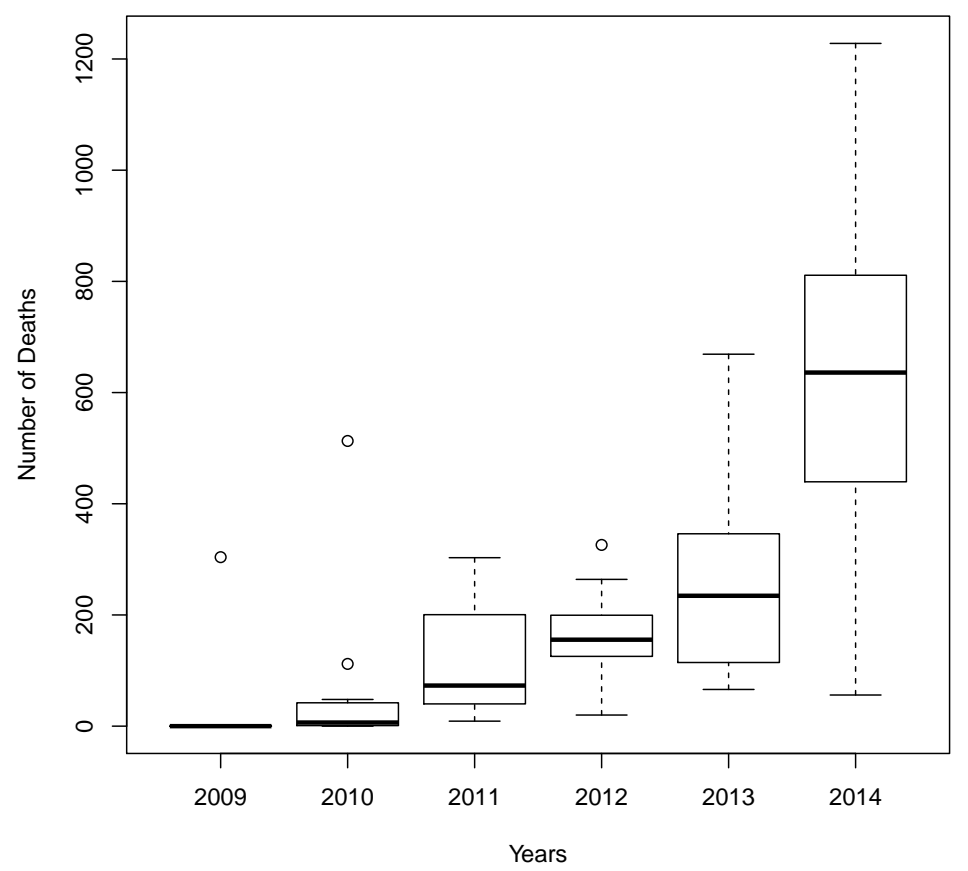

Fig. 1. Boxplots of the number of deaths resulted from Boko Haram attacks from January,2009-July,2014.

The proposed discrete distributions were constructed following [Nakagawa and Osaki (1975)] method of discretization. That is, given a continuous random variable $X$ with the survival function $S_{X}(x)=P(X \geq x)$, where $Y=[X]$ is a random variable denoting the largest integer part of $X$ which could be less or equal to $X$. Then the probability mass function of $Y$ is

$$
\begin{aligned}
P(Y=y) & =P(y \leq X<y+1) \\
& =P(X \geq y)-P(X \geq y+1) \\
& =S_{X}(y)-S_{X}(y+1)
\end{aligned}
$$

Thus, the discretized probability mass function $P(Y=y)$ arising from (1) is regarded as discrete concentration of probability density function of $X$ [Chakraborty and Chakravarty (2012)].

Journal home page: www.jafristatap.net 
Afuecheta E., Utazi C. and Ruiz A. P. African Journal of Applied Statictics, Vol. 3 (1), 2016, 101 - 120. A statistical analysis of fatalities resulting from Boko Haram activities.

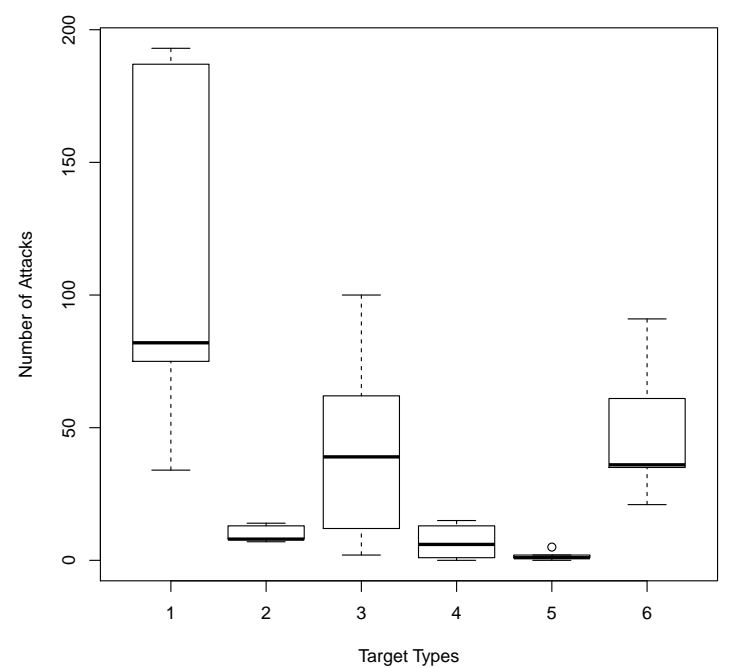

Fig. 2. Boxplots of the number of attacks versus the target types(Christian Community(1),Muslim Community(2), Government structures(3),Schools(4),Media/Medical(5) and others $(6))$.

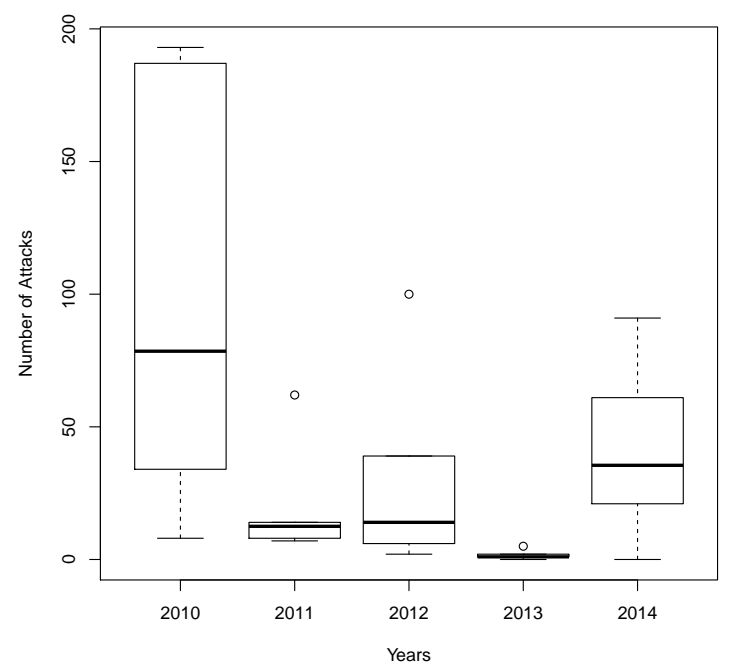

Fig. 3. Boxplots of the number of attacks versus years. 


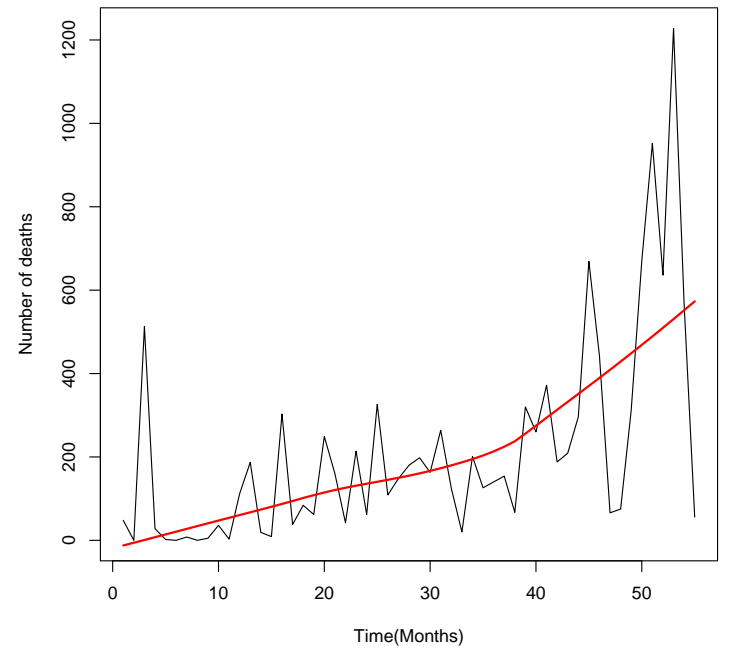

Fig. 4. Scatter plot of the number of deaths by Months.

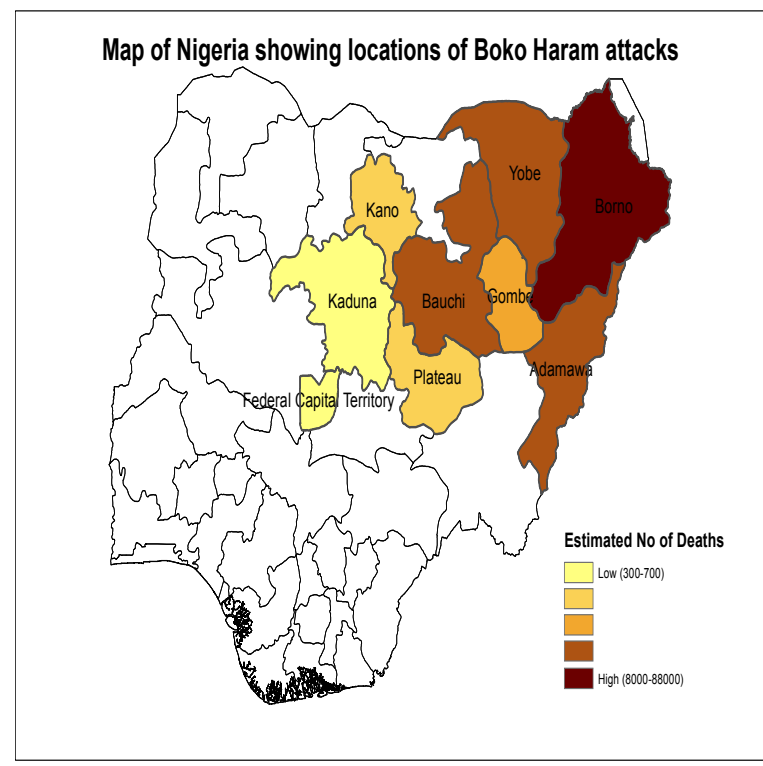

Fig. 5. Map of Nigeria showing mostly affected areas of Boko Haram attack

\subsection{Dagum distribuion}

Dagum distribution due to Dagum $(1975,1977,1980)$ with its probability mass function given by

$$
p(y)=\left[1+\left(\frac{y+1}{b}\right)^{-a}\right]^{-c}-\left[1+\left(\frac{y}{b}\right)^{-a}\right]^{-c}
$$

Journal home page: www.jafristatap.net 
where $a>0$, first shape parameter, $b>0$, the scale parameter, $c>0$, the second shape parameter, and $y=0,1, \cdots$.

\subsection{Schabe distribution}

Schabe distribution due to Schabe (1994) with its probability mass function given by

$$
p(x)=\frac{(1+\gamma)(y+1)}{(y+1)+\gamma \theta}-\frac{(1+\gamma) y}{y+\gamma \theta}
$$

where $0<\gamma<1$, the first scale parameter, $\theta>0$, the second scale parameter, and $y=$ $0,1, \cdots$.

\section{3. $L o g-\log$ distribution}

Loglog distribution due to Pham (2002) with its probability mass function given by

$$
p(y)=\exp \left[1-\lambda^{y^{\alpha}}\right]-\exp \left[1-\lambda^{(y+1)^{\alpha}}\right]
$$

where $\alpha>0$, the scale parameter, $\lambda>1$, the shape parameter, and $y=0,1, \cdots$.

\subsection{Log-logistic distribution}

$$
p(y)=\frac{(y+1)^{\beta}}{\alpha^{\beta}+(y+1)^{\beta}}-\frac{y^{\beta}}{\alpha^{\beta}+y^{\beta}}
$$

where $\alpha>0$, the scale parameter, $\beta>0$, the shape parameter, and $y=0,1, \cdots$.

\subsection{Lognormal distribution}

$$
p(y)=\Phi\left(\frac{\log (y+1)-\mu}{\sigma}\right)-\Phi\left(\frac{\log y-\mu}{\sigma}\right)
$$

where $\Phi$ is the cumulative distribution function of the standard normal random variable, $-\infty<\mu<\infty$, the location parameter, $\sigma>0$, the scale parameter, and $y=0,1, \cdots$.

\subsection{Generalized Pareto distribution}

Generalized Pareto distribution due to Pickands (1975) with its probability mass function given by

$$
p(y)=\left(1+\frac{\xi y}{\sigma}\right)^{-\frac{1}{\xi}}-\left(1+\frac{\xi(y+1)}{\sigma}\right)^{-\frac{1}{\xi}}
$$

where $\sigma>0$, the scale parameter, $\xi>0$, the shape parameter, and $y=0,1, \cdots$. 
Afuecheta E., Utazi C. and Ruiz A. P. African Journal of Applied Statictics, Vol. 3 (1), 2016, 101 - 120. A statistical analysis of fatalities resulting from Boko Haram activities.

\subsection{Fréchet distribution}

Fréchet distribution due to Fréchet (1927) with its probability mass function given by

$$
p(y)=\exp \left[-\left(\frac{\sigma}{y+1}\right)^{\xi}\right]-\exp \left[-\left(\frac{\sigma}{y}\right)^{\xi}\right]
$$

where $\sigma>0$, the scale parameter, $\xi>0$, the shape parameter, and $y=0,1, \cdots$.

\subsection{Inverse exponentiated exponential distribution}

Inverse exponentiated exponential distribution due to Ghitany et al. (2013) with its probability mass function given by

$$
p(y)=\left[1-\exp \left(-\frac{\lambda}{y}\right)\right]^{\alpha}-\left[1-\exp \left(-\frac{\lambda}{y+1}\right)\right]^{\alpha}
$$

where $\alpha>0$, the shape parameter, $\lambda>0$, the scale parameter, and $y=0,1, \cdots$.

\subsection{Chen distribution}

Chen distribution due to Chen (2000) with its probability mass function given by

$$
p(y)=\exp \left[\lambda-\lambda \exp \left(y^{\beta}\right)\right]-\exp \left[\lambda-\lambda \exp \left((y+1)^{\beta}\right)\right]
$$

where $\beta>0$, the shape parameter, $\lambda>0$, the scale parameter, and $y=0,1, \cdots$.

\subsection{Nakagami distribution}

Nakagami distribution due to Nakagami (1960)with its probability mass function given by

$$
p(y)=Q\left(m, \frac{m y^{2}}{a}\right)-Q\left(m, \frac{m(y+1)^{2}}{a}\right)
$$

where $a>0$, the scale parameter, $m>0$, the shape parameter, $y=0,1, \cdots$ and $Q(a, m)=$ $\frac{1}{\Gamma(a)} \int_{m}^{\infty} t^{a-1} \exp (-t) d t$ is the regularized complementary incomplete gamma function.

\subsection{Poisson distribution}

Poisson distribution is a known and popular distribution with its probability mass function given by

$$
p(y)=\frac{\lambda^{y} \exp (-\lambda)}{y !}
$$

where $\lambda>0$, the rate parameter, and $y=0,1, \cdots$. 
Afuecheta E., Utazi C. and Ruiz A. P. African Journal of Applied Statictics, Vol. 3 (1), 2016, 101 - 120. A statistical analysis of fatalities resulting from Boko Haram activities.

\section{Results and discussion}

The eleven discrete distributions in Section 3 were fitted to the number of deaths resulting from Boko Haram attacks in Nigeria. The method of maximum likelihood was used.The parameter estimates and their respective standard errors are given in Table 1, while the log-likelihood values, the Akaike information criterion (AIC)values, the Bayesian information criterion (BIC)values, the consistent Akaike information criterion (CAIC) values, the corrected Akaike information(AICc)values and the Hannan-Quinn criterion (HQC) values for all the fitted distributions are presented in Table 2. Note that the estimated standard errors of the MLE are the square roots of the diagonal elements of the inverse of the observed Fisher Information matrix.

We observe that the discrete Chen distribution gives the smallest values for the negative log-likelihood, the AIC, the BIC, the CAIC, the AICc and HQC. The discrete log-log distribution gives the second smallest values for the AIC, the BIC, the CAIC, the AICc, the HQC with third smallest value for the negative log-likelihood. The discrete Nakagami distribution gives the largest values for the negative log-likelihood, the AIC, the BIC, the CAIC, the AICc, and the HQC among the two parameters distributions used. In all,the discrete Chen distribution gives the least values for the negative log-likelihood, the AIC, the $\mathrm{BIC}$, the CAIC, the AICc, and the HQC and such becomes the best fitting distribution. The three-parameter distribution (Schabe) as well as the one-parameter distribution (Poisson), gives the largest values for the log-likelihood, the AIC, the BIC, the CAIC, the AICc and HQC. We test the goodness of the fit of the discrete Chen distribution. This can be accessed by various means. The Kolmogorov-Smirnov statistic and Anderson and Darling test statistic (Anderson and Darling, 1954) were used. Basically, these two tests concisely express the difference between the fitted $c d f, \widehat{F}$ and the empirical $c d f, F_{e}$. Specifically, the Anderson-Darling test, $\mathrm{AD}=\left(F_{e}(y)-\widehat{F}(y)\right)^{2}$ is the weighted average of the squared differences, whereas the Kolmogorov-Smirnov test, $\mathrm{KS}=\sup _{y}\left[\left|F_{e}(y)-\widehat{F}(y)\right|\right]$ is the largest distance between $F_{e}(y)$ and $\widehat{F}(y)$.

The Kolmogorov-Smirnov gives a $p$-value of 0.345 , while the Anderson and Darling test gives 0.928. These $p$-values confirm that the discrete Chen distribution adequately described the data.

\subsection{Time effect on number of fatalities}

The purpose of this section to investigate how the number of fatalities (deaths) varies with time. We shall assume that the parameters (shape and scale parameters) of discrete Chen distribution increase linearly with time. The choice of linearity is based on the scatter plot of the data. See Fig 4. Also, [Mann (1945)] non-parameteric test for trend gave the $p$-value of $=6.5989 \times 10^{-8}$ which further confirms our observation as well as choice of linearity. Hence we fitted the following linear models:

Model A: $\lambda=\exp (\psi), \beta=\exp (\zeta)$

Model B: $\lambda=\exp (\psi), \beta=\exp (\zeta+\delta \times M)$

Model C: $\lambda=\exp (\psi+\delta \times M), \beta=\exp (\zeta)$

Model D: $\lambda=\exp (\psi+\delta \times M), \beta=\exp (\zeta+\delta \times M)$

Journal home page: www.jafristatap.net 
Model E: $\lambda=\exp (\psi+\zeta \times M), \beta=\exp (\psi+\delta \times M)$

Model F: $\lambda=\exp (\psi+\zeta \times M), \beta=\exp (\delta+\eta \times M)$

where $\mathrm{M}$ represents time in months in our model

Model $\mathrm{A}$ is a two-parameter model with $\operatorname{scale}(\lambda)$ and $\operatorname{shape}(\beta)$ parameters totally independent of time. Model B is a three-parameter model with shape $(\beta)$ parameter assumed to vary linearly with time but scale $(\lambda)$ parameter remains fixed. Model $\mathrm{C}$ is a three-parameter model with scale $(\lambda)$ parameter assumed to vary linearly with time but shape $(\beta)$ parameter remains fixed. Model D is a three-parameter model with both shape $(\beta)$ and scale $(\lambda)$ parameter assumed to vary linearly with time with common slope. Model $\mathrm{E}$ is a three-parameter model with both shape $(\beta)$ and scale $(\lambda)$ parameters assumed to vary linearly with time with common intercept. Model $\mathrm{F}$ is a four-parameter model with both shape $(\beta)$ and scale $(\lambda)$ parameters assumed to vary linearly with time with different intercept and slope. To preserve the positivity of $\lambda$ and $\beta$, the regression functions are exponentiated as given in models A-F.

Each of the six models based on the best fitting discrete distribution is fitted using the method of maximum likelihood. We employ the standard ratio test by [Cox and Hinkley (1974)], which is a popular criterion for comparing models that are hierarchically nested to discriminate between the nested models. That is, we choose the best fitting model by determining if the difference in the likelihood ratio scores of the competing models are statistically significant.

The parameter estimates, the standard errors and the log-likelihood values for Models A-F are given in Table 3. Finally we use the AIC, the BIC, the CAIC, the AICc and the HQC values to select the overall best model among the competing models and the resulting values are presented in Table 4.

We see that the likelihood ratio test shows that neither of the nested models improves significantly. However, Model $\mathrm{F}$ gives the smallest values for the negative log-likelihood, the $\mathrm{AIC}$, the AICc and the HQC; therefore making it the best fitting model with significant trend in both the scale and shape parameters. The Model E is the second best fitting model with the smallest values for the BIC and CAIC.

For the scale parameter in our best fitting model, the number of deaths due to Boko Haram attacks shows an increase in trend. That is, for every unit increase in the number of months, the scale (variability) of the distribution of the number of deaths due to Boko Haram attacks increases by a factor of $\exp (0.003)$. This confirms the claim that Boko Haram activities is rapidly progressing, becoming more sophisticated, coordinated, menacingly daring and seemingly genocidal [Cook (2011)]; [Thomson (2012)] and [Agbiboa (2014)]. As evidenced by the change in dynamics of attacks and its operational ability which has resulted to a surge in the number of deaths recently, Boko Haram is probably receiving local/international training as well as assistance in form of funding.

In addition, as noted by some analysts, the collaboration of Boko Haram with other global terror organizations (such as Al-Qaeda, Al-Shabaab, Al-Qaeda in the Islamic Maghreb (AQIM), Al-Qaeda in the Arabian Peninsula (AQAP), etc ) is increasing their rate of dispersion and attacking prowess. For example,[McCaul et al. (2013)] argued that "the relationship between AQIM and Boko Haram has also influenced the adoption of kidnapping as a source of income, netting the group millions of dollars from ransoms. AQIM is fiscally

Journal home page: www.jafristatap.net 
Afuecheta E., Utazi C. and Ruiz A. P. African Journal of Applied Statictics, Vol. 3 (1), 2016, 101 - 120. A statistical analysis of fatalities resulting from Boko Haram activities.

\begin{tabular}{lllllll}
\hline $\begin{array}{l}\text { Discrete } \\
\text { distributions }\end{array}$ & $-\ln L$ & AIC & BIC & CAIC & AICc & HQC \\
\hline Dagum & 345.31 & 696.62 & 702.64 & 705.64 & 697.09 & 692.06 \\
Schabe & 705.95 & 1415.91 & 1415.39 & 1417.39 & 1416.14 & 1412.87 \\
Log-log & 345.49 & 694.99 & 694.47 & 696.47 & 695.22 & 691.95 \\
Log logistic & 353.56 & 711.12 & 710.60 & 712.60 & 711.35 & 708.08 \\
Lognormal & 356.10 & 716.10 & 715.68 & 717.68 & 716.43 & 713.16 \\
Generalized Pareto & 349.35 & 702.70 & 702.18 & 704.18 & 702.93 & 699.66 \\
Fréchet & 369.08 & 742.16 & 741.64 & 743.64 & 742.39 & 739.12 \\
Inv. exponentiated. exponential & 381.35 & 766.71 & 766.19 & & & \\
Chen & 345.00 & 694.02 & 693.50 & 695.50 & 694.25 & 690.98 \\
Nakagami & 666.24 & 1336.48 & 1335.96 & & & \\
Poisson & 6553.57 & 13109.14 & 13108.88 & 13109.88 & 13109.25 & 1307.62 \\
\hline
\end{tabular}

Table 1. Fitted distributions, log-likelihood values and selection criteria.

sound and has an arsenal of weapons at its disposal that has proven useful to Boko Haram". Nevertheless, the upheaval in Libya with its store houses of weapons, and ties with AQIM may provide Boko Haram with opportunities. However, ties with the two groups seem to be limited to training and possibly resources [Thomson (2012)]. [Leigh (2011)] argued that aside training, "the partnership between AQIM and Boko Haram can also mean an increase in cash flow for Boko Haram, which currently makes most of its money...by robbing banks throughout the Muslim northern half of the country". All these confirm that the increasing trend of the scale of the distribution of the number of deaths due to the Boko Haram's menace could be attributed to the increase in sphere and range of the sect's activities as well collaborations.

For the shape parameter, the number of deaths due to Boko Haram attacks shows a decrease in trend. That is for every unit increase in the number of months, the shape of the distribution of the number of deaths due to Boko Haram attacks decreases by a factor $\exp (-0.064)$.

\subsection{Prediction}

We make some predictions using our best fitting model in the penultimate Section 4.1. The predictions are the likely number of deaths into the first half of 2015. This holds provided that the Boko Haram's activities remains unchecked with the current trend of attack. Also, the predictions comprise of the pth percentiles (median, 95th, 99th) as presented in Table 5.

\section{The distributional Properties of the best fitting distribution}

From the results of the analysis, it is seen that the discrete Chen distribution was the best performing distribution based on the selection criteria (AIC, BIC, CAIC, AICc and HQC ) used. Here we give some of the distributional properties of the discrete Chen distribution. As a discretized class of continuous distribution often used in survival analysis, it is hoped that these properties will be useful for anyone interested in applying the discrete Chen 
Afuecheta E., Utazi C. and Ruiz A. P. African Journal of Applied Statictics, Vol. 3 (1), 2016, 101 - 120. A statistical analysis of fatalities resulting from Boko Haram activities.

\begin{tabular}{lll}
\hline Discrete & \multicolumn{1}{c}{ Parameter estimates } & Standard errors \\
\hline Distributions & $\widehat{a}=2.838, \widehat{b}=454.793, \widehat{c}=0.192$ & $0.853, \quad 115.353, \quad 0.077$ \\
Schabe & $\widehat{\gamma}=0.008, \widehat{\theta}=2.000$ & $0.001,0.286$ \\
Log-log & $\widehat{\alpha}=0.555, \widehat{\lambda}=1.037$ & $0.067,0.016$ \\
Log logistic & $\widehat{\alpha}=109.836, \widehat{\lambda}=1.032$ & $24.504,0.122$ \\
Lognormal & $\widehat{\mu}=4.451, \widehat{\sigma}=1.819$ & $0.246,0.182$ \\
Generalized Pareto & $\widehat{\sigma}=175.232, \widehat{\xi}=0.186$ & $39.837,0.185$ \\
Fréchet & $\widehat{\sigma}=32.463, \widehat{\xi}=0.461$ & $10.177,0.045$ \\
Inv. exponentiated exponential & $\widehat{\alpha}=0.307, \widehat{\lambda}=3.870$ & $0.048,1.133$ \\
Chen & $\widehat{\beta}=0.231, \widehat{\lambda}=0.033$ & $0.012,0.010$ \\
Nakagami & $\widehat{a}=50.000, \widehat{m}=6.034 \times 10^{-4}$ & $8.528 \times 10^{-3}, 5.901 \times 10^{-4}$ \\
Poisson & $\widehat{\lambda}=213.145$ & 1.969 \\
\hline
\end{tabular}

Table 2. Fitted models, parameter estimates and standard errors.

\begin{tabular}{llllcc}
\hline Models & $\widehat{\psi}(\mathrm{se})$ & $\widehat{\zeta}(\mathrm{se})$ & $\widehat{\delta}(\mathrm{se})$ & $\widehat{\eta}(\mathrm{se})$ & $-\ln L$ \\
\hline $\mathrm{A}$ & $-3.423(0.309)$ & $-1.467(0.051)$ & - & - & 345.009 \\
$\mathrm{~B}$ & $-3.648(0.326)$ & $-1.286(0.054)$ & $-0.004(0.001)$ & - & 339.323 \\
$\mathrm{C}$ & $-2.607(0.335)$ & $-1.370(0.046)$ & $-0.042(0.008)$ & - & 333.0641 \\
$\mathrm{D}$ & $-3.537(0.330)$ & $-1.292(0.053)$ & $-0.004(0.001)$ & - & 338.541 \\
$\mathrm{E}$ & $-1.542(0.046)$ & $-0.075(0.011)$ & $0.005(0.001)$ & & 332.428 \\
$\mathrm{~F}$ & $-1.461(0.082)$ & $0.003(0.002)$ & $-2.033(0.467)$ & $-0.064(0.015)$ & 331.839 \\
\hline
\end{tabular}

Table 3. Parameter estimates, standard errors and log-likelihood values, for the fit of Models A-F.

\begin{tabular}{lllllll}
\hline Models & & & & & & \\
& $-\ln L$ & AIC & BIC & CAIC & AICc & HQC \\
\hline A & 345.0093 & 694.0180 & 693.4987 & 695.4987 & 694.2488 & 690.9805 \\
B & 339.3234 & 684.6460 & 683.8671 & 686.8671 & 685.1166 & 680.0898 \\
C & 333.0641 & 672.1282 & 671.3493 & 674.3493 & 672.5988 & 667.5720 \\
D & 338.5411 & 683.0820 & 682.3031 & 685.3031 & 683.5525 & 678.5258 \\
E & 332.4276 & 676.8560 & 670.0771 & 673.0771 & 677.3266 & 666.2998 \\
F & 331.8391 & 671.6780 & 670.6395 & 674.6395 & 672.4780 & 665.6031 \\
\hline
\end{tabular}

Table 4. Fitted models, log-likelihood values and selection criteria.

distribution in reliability analysis. For instance, one may be interested in the failure time of a device when measurement are taken in discrete manner, such as, the number of cycles before the failure of a device, airplane tyre failure, computer failure, etc [Roy (2004)]. Given a continuous random variable $Y$ which follows Chen distribution with the $C D F$

$$
F(y)=1-\exp \left(\lambda-\lambda \exp (y)^{\beta}\right)
$$


Afuecheta E., Utazi C. and Ruiz A. P. African Journal of Applied Statictics, Vol. 3 (1), 2016, 101 - 120. A statistical analysis of fatalities resulting from Boko Haram activities.

\begin{tabular}{llll}
\hline Year & Median & $95 t h$ & $99 t h$ \\
\hline 2014 & 214 & 1231 & 1904 \\
2014 & 227 & 1271 & 1954 \\
2014 & 240 & 1310 & 2003 \\
2014 & 253 & 1349 & 2052 \\
2014 & 267 & 1388 & 2101 \\
2015 & 281 & 1427 & 2149 \\
2015 & 295 & 1466 & 2196 \\
2015 & 309 & 1504 & 2242 \\
2015 & 324 & 1543 & 2289 \\
2015 & 339 & 1581 & 2334 \\
2015 & 355 & 1618 & 2378 \\
\hline
\end{tabular}

Table 5. Fitted models, log-likelihood values and selection criteria.

where $\beta>0$, the shape parameter, $\lambda>0$, the scale parameter, and $y>0$. Following (1), the discrete Chen mass function is

$$
\begin{aligned}
p(y) & =\exp \left[\lambda-\lambda \exp \left(y^{\beta}\right)\right]-\exp \left[\lambda-\lambda \exp \left((y+1)^{\beta}\right)\right] \\
& =\exp -\lambda\left[\exp \left(y^{\beta}-1\right)\right]-\exp -\lambda\left[\exp \left((y+1)^{\beta}-1\right)\right]
\end{aligned}
$$

alternatively, if $\gamma=\exp (-\lambda)$ then

$$
\begin{aligned}
p(y) & =\gamma^{\exp (y)^{\beta}-1}-\gamma^{\exp (y+1)^{\beta}-1} \\
& =\gamma^{-1}\left[\gamma^{\exp (y)^{\beta}}-\gamma^{\exp (y+1)^{\beta}}\right]
\end{aligned}
$$

where $\quad 0<\gamma<1, \beta>0$, and $\quad y=0,1, \cdots$

Hence, the $C D F$ of (3) is given by

$$
F(x)=1-\gamma^{\exp (y+1)^{\beta}-1}
$$

with the survival function given by

$$
S(y)=\gamma^{\exp (y+1)^{\beta}-1}
$$

The failure rate(hazard function), FR is given by

$$
\begin{aligned}
r(y) & =\frac{p(y)}{S(y)} \\
& =-1+\gamma^{\exp (y)^{\beta}-\exp (y+1)^{\beta}}
\end{aligned}
$$

with its cumulative hazard function given by

$$
H(y)=y-\sum_{i=1}^{y} \gamma^{\exp (i+1)^{\gamma}-\exp (i)^{\gamma}}
$$


Note here that the discrete Chen distribution has an increasing failure rate(IFR). Evidently, if we define a conditional survival probability function, $S\left(t_{0} / t\right)$ which decreases over time $t$, such that, $S\left(t_{0} / t\right)=\frac{S\left(t+t_{0}\right)}{S(t)}$ is decreasing in time $t$ for every $t_{0}$, where $t_{0}$ denotes the time before the present time. Clearly,

$$
\lim _{t_{0} \rightarrow 0}\left[\frac{1}{t_{0}}\left(1-\frac{S\left(t+t_{0}\right)}{S(t)}\right)\right]=\lim _{t_{0} \rightarrow 0}\left[\left(\frac{S(t)-S\left(t+t_{0}\right)}{t_{0} S(t)}\right)\right]=K(t)
$$

increases in $t$ provided that $t_{0}$ approaches zero. As such,

$$
r(y)=1-\gamma^{\exp (y+1)^{\beta}-\exp (y)^{\gamma}}
$$

is an increasing failure rate provided $y \geq 0$. See Fig 7 . for the plots of the failure rate of the discrete Chen distribution.

The second failure rate $(\mathrm{SRF})$ is given by

$$
\begin{aligned}
r(y) & =\log \left[\frac{S(y)}{S(y+1)}\right] \\
& =\log \left[\gamma^{\exp (y)^{\beta}-\exp (y+1)^{\beta}}\right] \\
& =\left[\exp (y)^{\beta}-\exp (y+1)^{\beta}\right] \log \gamma
\end{aligned}
$$

\subsection{Parameter Estimation by maximum likelihood}

Suppose $y_{1}, \cdots, y_{n}$ are samples from the discrete Chen distribution with vector of unknown parameters, $\boldsymbol{\Psi}=\left(\psi_{1}, \psi_{2}\right)=(\gamma, \beta)$. Then, the log-likelihood function is given by

$$
\log L\left(\Psi ; y_{i}\right)=-n \log \gamma+\sum_{y=1}^{n} \log \left(\gamma^{\exp (y)^{\beta}}-\gamma^{\exp (y+1)^{\beta}}\right)
$$

The derivatives of (9) with respect to $\gamma$ and $\beta$ are given below by equations (10) and (11) respectively

$$
\begin{aligned}
& \frac{\partial \log L\left(\boldsymbol{\Psi} ; y_{i}\right)}{\partial \gamma}=-\frac{n}{\gamma}+\sum_{i=1}^{n} \frac{e^{y(i)^{\beta}} \gamma^{-1+e^{y(i)^{\beta}}}-e^{\left(1+y_{i}\right)^{\beta}} \gamma^{-1+e^{\left(1+y_{i}\right)^{\beta}}}}{\gamma^{e^{y_{i}^{\beta}}}-\gamma^{e^{\left(1+y_{i}\right)^{\beta}}}} \\
& \frac{\partial \log L\left(\boldsymbol{\Psi} ; y_{i}\right)}{\partial \beta}=\sum_{i=1}^{n} \frac{e^{y(i)^{\beta}} \gamma^{e^{y_{i}^{\beta}}} \log (\gamma) \log \left(y_{i}\right) y(i)^{\beta}}{\gamma^{y^{\beta}}-\gamma^{e^{\left(1+y_{i}\right)^{\beta}}}} \\
&-\frac{e^{\left(1+y_{i}\right)^{\beta}} \gamma^{e^{\left(1+y_{i}\right)^{\beta}}} \log (\gamma) \log \left(1+y_{i}\right)\left(1+y_{i}\right)^{\beta}}{\gamma^{e^{y_{i}^{\beta}}}-\gamma^{e^{\left(1+y_{i}\right)^{\beta}}}}
\end{aligned}
$$

Consequently, the MLEs of $\hat{\gamma}$ and $\hat{\beta}$ are the simultaneous solutions of 
Afuecheta E., Utazi C. and Ruiz A. P. African Journal of Applied Statictics, Vol. 3 (1), 2016, 101 - 120. A statistical analysis of fatalities resulting from Boko Haram activities.

$\frac{\partial \log L\left(\boldsymbol{\Psi} ; y_{i}\right)}{\partial \gamma}=0$ and $\frac{\partial \log L\left(\boldsymbol{\Psi} ; y_{i}\right)}{\partial \beta}=0$. Any of the iterative technique such quasi-Newton algorithm could be used to solve the equations (10) and (11)

However, to obtain the confidence intervals for $\gamma$ and $\beta$, the observed information matrix is computed by taking the second order partial derivatives of the log-likelihood function, that is

$$
\begin{aligned}
& \frac{\partial^{2} \log L\left(\boldsymbol{\Psi} ; y_{i}\right)}{\partial \gamma^{2}}=-I_{11}= \\
& \frac{n}{\gamma^{2}}+\sum_{i=1}^{n}\left(\frac{\mathcal{D}_{1}-e^{\left(1+y_{i}\right)^{\beta}}\left(-1+e^{\left(1+y_{i}\right)^{\beta}}\right) \gamma^{-2+e^{\left(1+y_{i}\right)^{\beta}}}}{\gamma^{e^{y_{i}^{\beta}}}-\gamma^{e^{\left(1+y_{i}\right)^{\beta}}}}\right. \\
& \left.-\frac{\left(e^{y_{i}^{\beta}} \gamma^{-1+e^{y_{i}^{\beta}}}-e^{\left(1+y_{i}\right)^{\beta}} \gamma^{-1+e^{\left(1+y_{i}\right)^{\beta}}}\right)^{2}}{\left(\gamma^{e^{y_{i}^{\beta}}}-\gamma^{e^{\left(1+y_{i}\right)^{\beta}}}\right)^{2}}\right)
\end{aligned}
$$

where $\mathcal{D}_{1}=e^{y_{i}^{\beta}}\left(-1+e^{y_{i}^{\beta}}\right) \gamma^{-2+e^{y_{i}^{\beta}}}$

$$
\begin{aligned}
& \frac{\partial^{2} \log L\left(\boldsymbol{\Psi} ; y_{i}\right)}{\partial \gamma \partial \beta}=-I_{12}= \\
& \sum_{i=1}^{n}\left(-\frac{1}{\left(\gamma^{e^{y_{i}^{\beta}}}-\gamma^{e^{\left(1+y_{i}\right)^{\beta}}}\right)^{2}} \mathcal{D}_{21} \times\right. \\
& \left(e^{y_{i}^{\beta}} \gamma^{e^{y_{i}^{\beta}}} \log (\gamma) \log \left(y_{i}\right) y_{i}^{\beta}-\mathcal{D}_{22}\right) \\
& +\frac{e^{y_{i}^{\beta}} \gamma^{-1+e^{y_{i}^{\beta}}} \log \left(y_{i}\right) y_{i}^{\beta}}{\gamma^{e^{y_{i}^{\beta}}}-\gamma^{e^{\left(1+y_{i}\right)^{\beta}}}}+\frac{e^{2 y_{i}^{\beta}} \gamma^{-1+e^{y_{i}^{\beta}}} \log (\gamma) \log \left(y_{i}\right) y_{i}^{\beta}}{\gamma^{e^{y_{i}^{\beta}}}-\gamma^{e^{\left(1+y_{i}\right)^{\beta}}}} \\
& -\frac{e^{\left(1+y_{i}\right)^{\beta}} \gamma^{-1+e^{\left(1+y_{i}\right)^{\beta}}} \log \left(1+y_{i}\right)\left(1+y_{i}\right)^{\beta}}{\gamma^{e^{y_{i}^{\beta}}}-\gamma^{e^{\left(1+y_{i}\right)^{\beta}}}}- \\
& \left.\frac{e^{2\left(1+y_{i}\right)^{\beta}} \gamma^{-1+e^{\left(1+y_{i}\right)^{\beta}}} \log (\gamma) \log \left(1+y_{i}\right)\left(1+y_{i}\right)^{\beta}}{\gamma^{e^{y_{i}^{\beta}}}-\gamma^{e^{\left(1+y_{i}\right)^{\beta}}}}\right)
\end{aligned}
$$




$$
\begin{aligned}
& \frac{\partial^{2} \log L\left(\Psi ; y_{i}\right)}{\partial \beta^{2}}=-I_{22}= \\
& \sum_{i=1}^{n}\left(-\frac{1}{\left(\gamma^{e^{y_{i}^{\beta}}}-\gamma^{e^{\left(1+y_{i}\right)^{\beta}}}\right)^{2}} \times\right. \\
& \left(e^{y_{i}^{\beta}} \gamma^{e^{y_{i}^{\beta}}} \log (\gamma) \log \left(y_{i}\right) y_{i}^{\beta}-\mathcal{D}_{31}\right)^{2} \\
& +\frac{e^{y_{i}^{\beta}} \gamma^{e^{y_{i}^{\beta}}} \log (\gamma) \log \left(y_{i}\right)^{2} y_{i}^{\beta}+e^{y_{i}^{\beta}} \gamma^{e^{y_{i}}} \log (\gamma) \log \left(y_{i}\right)^{2} y_{i}^{2 \beta}}{\gamma^{y^{\beta}}-\gamma^{e^{\left(1+y_{i}\right)^{\beta}}}} \\
& +\frac{e^{2 y_{i}^{\beta}} \gamma^{e^{y_{i}^{\beta}}} \log (\gamma)^{2} \log \left(y_{i}\right)^{2} y_{i}^{2 \beta}-\mathcal{D}_{32}}{\gamma^{e^{y_{i}^{\beta}}}-\gamma^{e^{\left(1+y_{i}\right)^{\beta}}}} \\
& -\frac{e^{\left(1+y_{i}\right)^{\beta}} \gamma^{e^{\left(1+y_{i}\right)^{\beta}}} \log (\gamma) \log \left(1+y_{i}\right)^{2}\left(1+y_{i}\right)^{2 \beta}}{\gamma^{y_{i}^{\beta}}-\gamma^{e^{\left(1+y_{i}\right)^{\beta}}}} \\
& -\frac{e^{2\left(1+y_{i}\right)^{\beta}} \gamma^{e^{\left(1+y_{i}\right)^{\beta}}} \log (\gamma)^{2} \log \left(1+y_{i}\right)^{2}\left(1+y_{i}\right)^{2 \beta}}{y^{\beta}}-\gamma^{e^{\left(1+y_{i}\right)^{\beta}}}
\end{aligned}
$$

where

$$
\begin{aligned}
& \mathcal{D}_{31}=e^{\left(1+y_{i}\right)^{\beta}} \gamma^{e^{\left(1+y_{i}\right)^{\beta}}} \log (\gamma) \log \left(1+y_{i}\right)\left(1+y_{i}\right)^{\beta} \\
& \mathcal{D}_{32}=e^{\left(1+y_{i}\right)^{\beta}} \gamma^{e^{\left(1+y_{i}\right)^{\beta}}} \log (\gamma) \log \left(1+y_{i}\right)^{2}\left(1+y_{i}\right)^{\beta} . \\
& \text { with } I(\Psi)=-\left(\begin{array}{ll}
I_{1,1} & I_{1,2} \\
I_{2,1} & I_{2,2}
\end{array}\right)
\end{aligned}
$$

The covariance matrix , $V$ is obtained as $V=\left[I_{\hat{\Psi}}\right]^{-1}=\left(\begin{array}{ll}V_{1,1} & V_{1,2} \\ V_{2,1} & V_{2,2}\end{array}\right)$

and the asymptotic distribution of the MLEs of the parameters converges to a bivariate normal random vector with zero means as

$$
\sqrt{n}\left[\left(\begin{array}{l}
\hat{\gamma} \\
\hat{\beta}
\end{array}\right)-\left(\begin{array}{l}
\gamma \\
\beta
\end{array}\right)\right]_{n \rightarrow \infty} \longrightarrow N_{2}\left[\left(\begin{array}{l}
0 \\
0
\end{array}\right),\left(\begin{array}{ll}
V_{\sigma_{11}} & V_{\sigma_{12}} \\
V_{\sigma_{21}} & V_{\sigma_{22}}
\end{array}\right)\right]
$$

Hence, the confidence interval for the parameters, $\hat{\gamma}, \hat{\beta}$ of the discrete Chen are given as

$$
\left(\hat{\gamma}-Z_{\frac{\nu}{2}} \sqrt{V_{11}}, \hat{\gamma}+Z_{\frac{\nu}{2}} \sqrt{V_{11}}\right) \quad \text { and } \quad\left(\hat{\beta}-Z_{\frac{\nu}{2}} \sqrt{V_{22}}, \hat{\beta}+Z_{\frac{\nu}{2}} \sqrt{V_{22}}\right) \text { respectively. }
$$

Following [Kemp (2004)] and [Krishna and Pundir (2009)], we obtain the moments of the discrete Chen distribution as follows 


$$
\begin{aligned}
m_{r}^{\prime} & =E\left(y^{r}\right)=\sum_{y=0}^{\infty} y^{r} p(y) \\
& =\sum_{y=0}^{\infty} y^{r}(S(y)-S(y+1)) \\
& =\sum_{y=1}^{\infty}\left(y^{r}-(y-1)^{r}\right) S(y) \leq \sum_{y=1}^{\infty} r y^{r-1} S(y)
\end{aligned}
$$

Therefore, from equation (15) is not difficult to see that the first four moments are

$$
\begin{gathered}
m_{1}^{\prime}=\gamma^{-1} \sum_{y=1}^{\infty} \gamma^{\exp (y)^{\beta}} \\
m_{2}^{\prime}=\gamma^{-1} \sum_{y=1}^{\infty}(2 y-1) \gamma^{\exp (y)^{\beta}} \\
m_{3}^{\prime}=\gamma^{-1} \sum_{y=1}^{\infty}\left(3 y^{2}-3 y+1\right) \gamma^{\exp (y)^{\beta}} \\
m_{4}^{\prime}=\gamma^{-1} \sum_{y=1}^{\infty}\left(4 y^{3}-6 y^{2}+4 y-1\right) \gamma^{\exp (y)^{\beta}}
\end{gathered}
$$




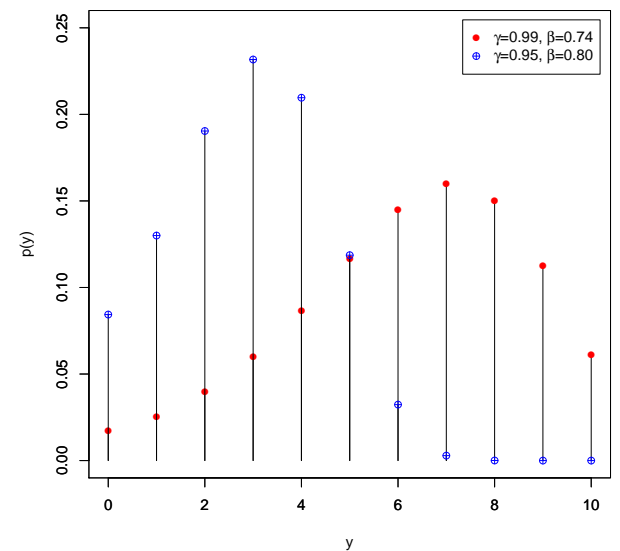

(a)

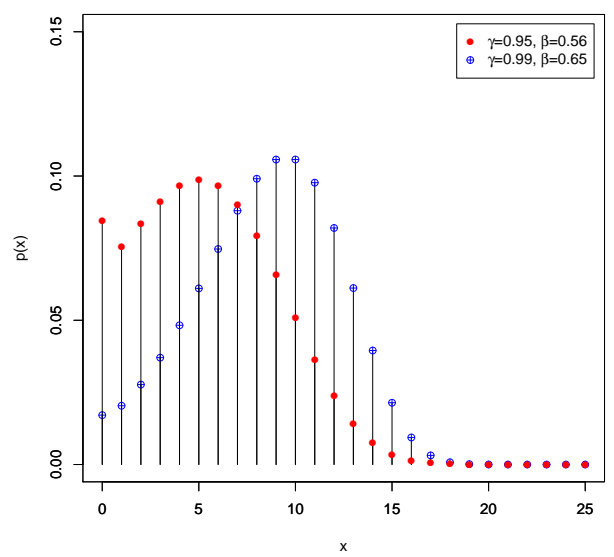

(c)

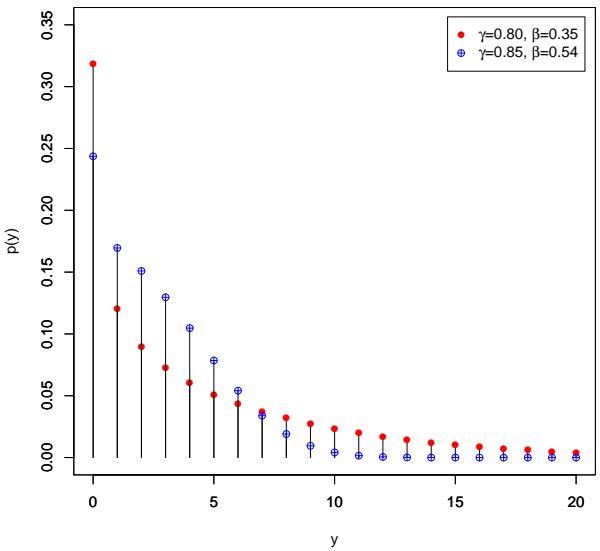

(b)

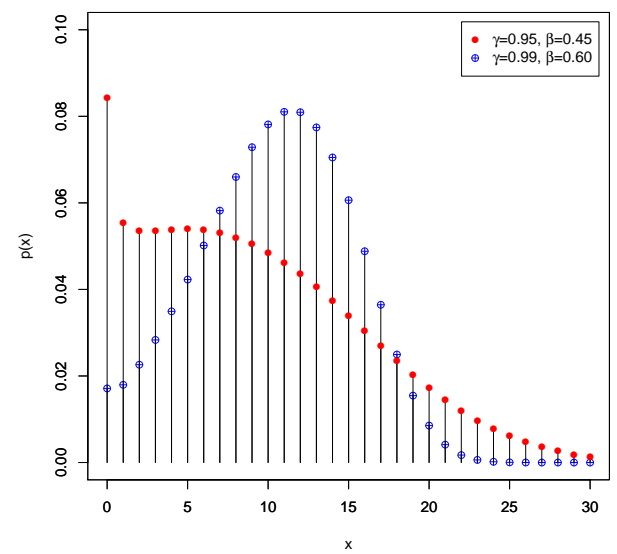

(d)

Fig. 6. PMFs of the discrete Chen distribution.

\section{Conclusions}

We have used eleven discrete distributions to analyze the number of deaths due to Boko Haram attacks in Nigeria. The fitted distributions are: the discrete Dagum distribuion, the discrete Schabe distribution, the discrete log-log distribution, the discrete log-logistic distribution, the discrete lognormal distribution, the discrete generalized Pareto distribution, the discrete Fréchet distribution, the discrete inverse exponentiated exponential distribution, the discrete Chen distribution, the discrete Nakagami distribution, and the Poisson distribution. The discrete Chen distribution significantly out-performed others as the best fitting 


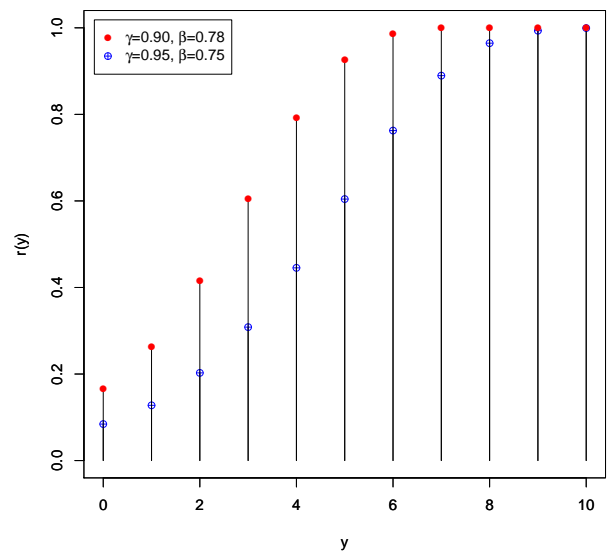

(a)

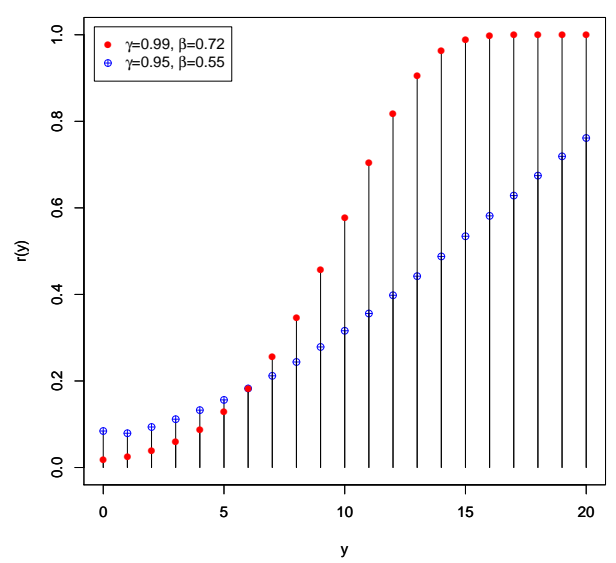

(c)

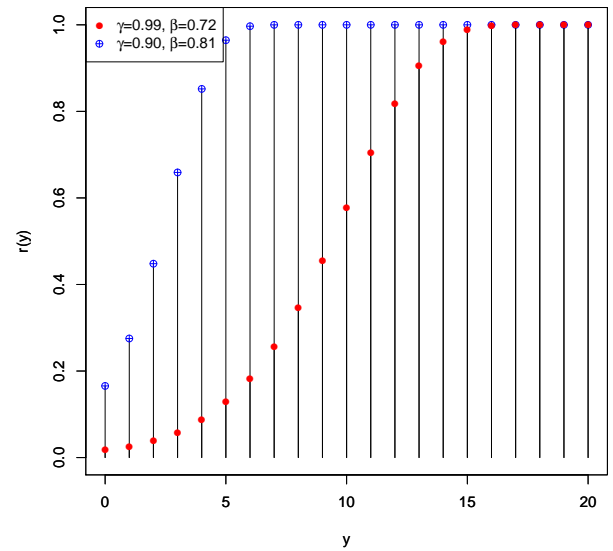

(b)

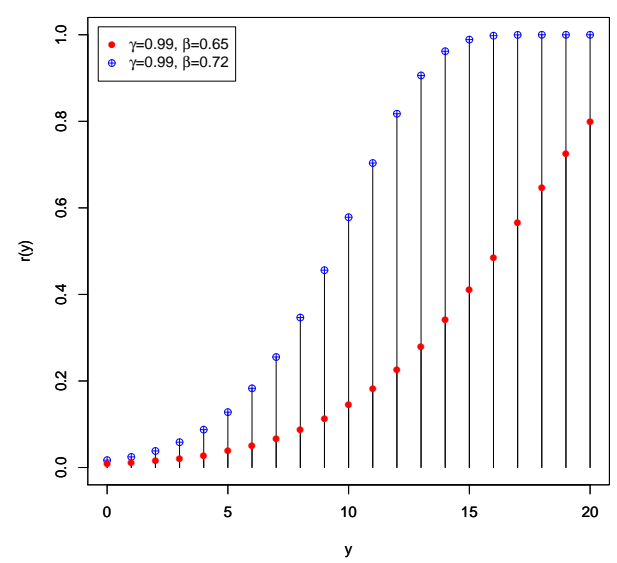

(d)

Fig. 7. Failure rate functions of the discrete Chen distribution.

distribution in terms of the negative log-likelihood values, the AIC, the BIC, the CAIC, the AICc, and the HQC values. Evidence of an increasing trend in the distribution of the number of deaths was shown. We have also given future predictions for possible number of deaths. The basic distributional properties of the Chen distribution are also calculated. 
Afuecheta E., Utazi C. and Ruiz A. P. African Journal of Applied Statictics, Vol. 3 (1), 2016, 101 - 120. A statistical analysis of fatalities resulting from Boko Haram activities.

\section{References}

Maiangwa, B. and Uzodike, U. O. (2012). The changing dynamics of Boko Haram terrorism, Report, Al Jazeera Centre for Studies, 1-6.

International Crisis Group, 2014. Curbing Violence in Nigeria (II): The Boko Haram Insurgency. Africa Report, 216, http://www.crisisgroup.org/.

Walker, A., 2012. Special report on Boko Haram. 2301 Constitution Ave,. United States Institute of Peace, www.usip.org.

Onapajo, H. and Uzodike, U. O. (2012). Boko Haram terrorism in Nigeria. African Security Review, vol. 21, (3), pp. 24-39.

Thomson, V. (2012). Boko Haram and Islamic fundamentalism in Nigeria. Global Security Studies, 3, issue 3,

Agbiboa, D. E., 2014. Peace at daggers drawn? Boko Haram and the state of emergency in Nigeria. Studies in Conflict and Terrorism, vol. 37, (1), pp. 41-67.

Adesoji, A., (2010). The Boko Haram uprising and islamic revivalism in Nigeria. Africa Spectrum, vol. 45 (2), pp. 95-108.

Aghedo, I., and Osumah, O., (2012). The Boko Haram uprising: How should Nigeria respond?. Third World Quarterly, vol. 33(52), 853-869.

Nadarajah, S., (2013). A statistical analysis of Iraq body counts. Quai Quant, DOI $10.1007 / s 11135-013-9971-9$

Nakagawa, T and Osaki, S.(1975). The discrete Weibull distribution. IEEE Transactions on Reliability, vol. 24(5), 300-301.

Chakraborty, S. and Chakravarty, D. (2012). Discrete gamma distributions: Properties and parameter estimations. Communication in Statistics-Theory and Methods, vol. 41(18), 3301-3324.

Mann, H. B.(1945). Nonparametric test against trend. Econometrica, 13, 245-259

Cox, D. R. and Hinkley, D. V. (1974). Theoretical Statistics. Chapman and Hall, London.

Cook, D. (2011). Boko Haram: A Prognosis. Retrieved from James A. Baker III Institute for Public Policy, Rice University website: http://bakerinstitute.org/publications/REL-pubCookBokoHaram-121611.pdf .

Agbiboa, D. E., (2014). Peace at daggers drawn? Boko Haram and the state of emergency in Nigeria. Studies in Conflict and Terrorism, vol. 37 (1), 41-67.

McCaul, M. T; Meehan, P. L. and Peter, T. K.(2013). Boko Haram: Growing threat to the US homeland. US house of representatives committee on homeland security. http://homeland.house.gov/sites/homeland.house.gov/files/documents/09-13-13-BokoHaram-Report.pdf

Leigh, K. (2011). Nigeria's Boko Haram: Al-Qaeda's new friend in Africa?. TIME.

Kemp, A. W. (2004) Class of Discrete Lifetime Distributions. Communication in StatisticsTheory and Methods, vol.33(12), 3069-3093 DOI:10.1081/STA-200039051

Krishna, H. and Pundir, P. S. (2009). Discrete Burr and discrete Pareto distributions. Statistical Methodology, 6, 177-188.

Johnson, N. L; Kotz, S. and Kemp, A. W.(1992). Univariate Discrete Distribution, 2nd ed. Wiley, New York.

Dilip Roy (2004) Discrete Rayleigh distribution IEEE Transactions on Reliability, vol. 53 (2), pp. $255-260$ 